Less can be more: How to make operations more flexible and robust with fewer resources

Çağrı Haksöz, Konstantinos Katsikopoulos, and Gerd Gigerenzer

Citation: Chaos 28, 063102 (2018); doi: 10.1063/1.5024259

View online: https://doi.org/10.1063/1.5024259

View Table of Contents: http://aip.scitation.org/toc/cha/28/6

Published by the American Institute of Physics 


\title{
Less can be more: How to make operations more flexible and robust with fewer resources
}

\author{
Çağrı Haksöz,, ${ }^{1, a)}$ Konstantinos Katsikopoulos, ${ }^{2, b)}$ and Gerd Gigerenzer ${ }^{3, c)}$ \\ ${ }^{1}$ Krannert School of Management, Purdue University 403 W. State St., West Lafayette, Indiana 47907, USA \\ and Indian School of Business, Gachibowli, Hyderabad, Telangana 500 111, India \\ ${ }^{2}$ Southampton Business School, University of Southampton, Highfield, Southampton SO17 1BJ, \\ United Kingdom \\ ${ }^{3}$ Max Planck Institute for Human Development, Harding Center for Risk Literacy Lentzeallee 94, \\ 14195 Berlin, Germany
}

(Received 30 January 2018; accepted 14 May 2018; published online 5 June 2018)

\begin{abstract}
We review empirical evidence from practice and general theoretical conditions, under which simple rules of thumb can help to make operations flexible and robust. An operation is flexible when it responds adaptively to adverse events such as natural disasters; an operation is robust when it is less affected by adverse events in the first place. We illustrate the relationship between flexibility and robustness in the context of supply chain risk. In addition to increasing flexibility and robustness, simple rules simultaneously reduce the need for resources such as time, money, information, and computation. We illustrate the simple-rules approach with an easy-to-use graphical aid for diagnosing and managing supply chain risk. More generally, we recommend a four-step process for determining the amount of resources that decision makers should invest in so as to increase flexibility and robustness. Published by AIP Publishing. https://doi.org/10.1063/1.5024259
\end{abstract}

In March 2011 a tsunami hit Japan. The production facility of Kureha, a company responsible for meeting approximately $\mathbf{7 0} \%$ of the worldwide demand of a polymer for manufacturing mobile-device batteries, was badly affected and could not deliver. As a consequence, Apple, Samsung, and others could not deliver either. With the benefit of hindsight, Apple and other original equipment manufacturers should have had more than one source for the polymer. So, how many suppliers should a manufacturer have? Two, three? The more the better? And how should they be chosen? The traditional approach to answering such questions is to set up and solve analytical models (e.g., models of optimization under constraints). But in global supply chains, the resources necessary for solving such models-such as adequate time and high-quality information-are often unattainable. Is there an alternative to complex models? The answer is yes. Simple rules of thumb can, under some conditions, provide better solutions than complex models to supply chain disruptions and a host of other challenging problems such as the allocation of wealth to financial assets, the analysis of customer base in order to target future advertising, or the prediction of bank failure, while simultaneously reducing the need for resources such as time, money, information, and computation. Simple rules can make operations more flexible as well as more robust. An operation is flexible when it responds adaptively to adverse events such as the Japanese tsunami. An operation is robust when it is little affected by adverse events in the first place. The concept of flexibility is known by managers, whereas the concept of robustness less so, and the

\footnotetext{
a)chaksoz@purdue.edu and cagri_haksoz@isb.edu

b)K.Katsikopoulos@soton.ac.uk

c) sekgigerenzer@mpib-berlin.mpg.de
}

two have not been related to each other. In sum, against the recent trend of focusing on resource-intensive models, the results presented herein suggest that there are good reasons for considering simple rules of thumb.

\section{INTRODUCTION}

In March 2011 a tsunami hit Japan. The production facility of Kureha, a company responsible for meeting approximately $70 \%$ of the worldwide demand of a polymer for manufacturing mobile-device batteries, was badly affected and could not deliver. As a consequence, Apple, Samsung, and others could not deliver either. With the benefit of hindsight, Apple and other original equipment manufacturers should have had more than one source for the polymer. So, how many suppliers should a manufacturer have? Two, three? The more the better? And how should they be chosen?

The traditional approach to answering such questions is to set up and solve analytical models (e.g., models of optimization). But in the context of global, dynamic, and uncertain supply chains, the resources necessary for solving such modelssuch as adequate time and high-quality information-may not always be attainable. Is there an alternative to complex models?

The answer is yes. Simple rules of thumb can, under some conditions, provide better solutions than complex models to supply chain disruptions and a host of other challenging problems such as the allocation of wealth to financial assets, the analysis of customer base in order to target future advertising, or the prediction of bank failure, while simultaneously reducing the need for resources such as time, money, information, and computation. Simple rules, if used intelligently, can help make operations, specifically supply chains, more flexible as well as more robust. 
In this paper, following a strategic decision making lens, we use the following definitions for flexibility and robustness. An operation is flexible when it responds adaptively to adverse events such as the Japanese tsunami. An operation is robust when it is little affected by adverse events in the first place. Previous research in supply chain management has widely studied the flexibility and robustness from different angles in the context of supply chain risk. (For an integrated review, see, for example, Haksöz (2013) and references therein). Inspired by the work of Rosenhead (2013), who shows that the robustness of an initial decision is a measure of flexibility maintained for future decision making, we illustrate that flexibility and robustness are related through the use of simple rules of thumb. To our knowledge, this intimate connection of flexibility and robustness of supply chains considering simple rules has not been addressed.

In Sec. II, we explain how "less can be more" in operational decisions, focusing on supply chain risk management. The results are summarized in an easy-to-use graphical aid for diagnosing and managing supply chain risk. In Sec. III, moving beyond the supply chain risk, we present empirical evidence on the relative performance of simple rules of thumb and more complex models on other decision problems, as well as general theoretical conditions under which the one outperforms the other (Gigerenzer et al., 2011). These results are summarized in a straightforward table. On the basis of this work, we recommend a four-step process for determining the amount of resources that decision makers should invest in so that they can make operations more flexible and robust. In the concluding section, we also discuss similar ideas on simple rules in other fields such as strategic management and systems thinking.

\section{LESS CAN BE MORE IN SUPPLY CHAIN RISK MANAGEMENT}

\section{A. How to construct a portfolio of suppliers?}

Dual sourcing in procurement is a widely accepted industry practice in various industries and organizations. Global telecommunications company Cisco uses dual sourcing. In the automotive industry, Freescale Semiconductor's Japan president David Uze says that after the shutdown of Japanese carmakers for months following the 2011 tsunami, "dual sourcing is industry policy now" (Greimel, 2014). Turkish tire manufacturer Brisa (a joint venture of Bridgestone Corporation and Sabanc1 Holding) uses a portfolio of two suppliers for critical raw materials in its global supply chain, one located nearby, in Europe or the Middle East, and another one far away, in the Far East (see Haksöz (2013) for details of this field study on supply chains that thrive in fragile contexts). Brisa supply chain director explains the operation of their dual sourcing rule:

"Our team examines each raw material and finds out whether it is supplied by a single supplier. If it is, then the next step is to see if it is a global or local supplier. For global suppliers, we look for substitutes. If there is an emergency, is it possible to use an unapproved supplier or can we shorten the approval procedure or bypass a part of it? We examine whether we can shift this material's procurement from European suppliers to other locations."

According to Brisa's supply chain director, dual sourcing allowed the company to deal with the disruptive effects of the Fukushima tsunami:

"I am glad to say that the impact was minimal. Surely Japanese facilities were affected. There was one particular material we were procuring from Japan. With the coordination of Bridgestone, we switched to a US supplier. We also did some production prescription changes and used a substitute material with the same specs in our production. In the end, since some of our raw materials sourced in the Far East may have disruptions, we use a dual sourcing strategy including a near source in addition to a far-away source" (Haksöz, 2013).

Location-based dual sourcing circumvents the common dilemma of manufacturers of how to choose between an onshore or near-shore and an offshore supplier. It may be the way to go for a company that operates a global supply chain with exposure to diverse risks spread across large geographic areas. Companies having a stable onshore or near-shore supplier, say in North America, Latin America, or Europe, can add an offshore supplier, for instance, in China, Eastern Europe, or the Middle East. In the words of the global head of procurement of another Turkish manufacturing company, Kordsa Global, which is a global market leader, producing industrial fabrics and reinforcement materials in nine countries:

"We prefer to have suppliers at different geographic areas such as one in Europe and one in the US or Asia. There are tax and customs benefits for such an arrangement, as well as proximity considerations. We can mitigate natural disaster risks better in such a portfolio, that is, when a hurricane hits the US shores, Europe is safe; likewise, when the Rhine River has water shortage issues in Europe, the American supplier will stay unaffected." (Haksöz, 2013)

One may expect dual sourcing to be advantageous in that the buyer avoids the cost and complexity of coordinating with too many suppliers while obtaining most of the benefits of multiple sourcing. This intuition has been corroborated by theoretical research by Tang and Tomlin (2008) and Allon and Van Mieghem (2010). Assuming that supplier costs can be modeled by a probability distribution (Bernoulli, uniform, or exponential), it has been shown analytically that the expected unit cost of ordering from multiple suppliers decreases with the number of suppliers and reaches an asymptote for a small number of suppliers. On the other hand, it is clear that coordination costs increase with the number of suppliers. Thus, the sum of these types of costs can be predicted to be minimum for a few, possibly approximately two, suppliers. In sum, a little flexibility in the number of suppliers may bring most of the value that can be attained in the supply chain (Tang and Tomlin, 2008).

Supplier portfolios can also be constructed in more sophisticated ways than by considering just one attribute such as supplier location in the previous examples. In dual 
sourcing, one supplier can be chosen for being able to provide at low cost and another one chosen for being able to deliver at short notice. For instance, a $\$ 10$ billion high-tech U.S. manufacturer of wireless transmission components had two assembly plants, one in China and another in Mexico (Allon and Van Mieghem, 2010). The Chinese plant had lower costs, but ocean transportation made its delivery times five to ten times longer than those from Mexico. With highly uncertain product demand-coefficients of variation of monthly demand for some products reached 1.25-sole sourcing was unattractive. Mexico was too expensive and China too unresponsive. Inspired by such problems, a portfolio has been proposed where the stable base demand-say, three quarters of all demand-is handled by a low-cost/lowspeed supplier, and the remaining unexpected surge demand is met by a high-cost/high-speed supplier. It has been shown analytically that a low-cost/low-speed supplier together with a high-cost/high-speed supplier generate almost all of the theoretically expected value of a portfolio of all known suppliers (Fu et al., 2010).

In sum, a simple approach is viable: Supplier portfolio construction entails identifying only the one supplier with the lowest cost and the one with the highest speed (which, in many cases, would be a supplier which is located nearby). That is, a more feasible and less resource-intensive task than using an optimization model to identify suppliers in the context of high uncertainty. In other words, a small amount of flexibility in supplier portfolio can lead to a great deal of robustness enhancing the value and reducing the impact of disruption. This is related to Rosenhead's (2013) point that the robustness of an initial decision is an operational measure of the flexibility maintained for future decision-making.

The point that a bit of flexibility can lead to a lot of robustness is generally often true of complex adaptive systems when they are self-organized critical [see Bak et al. (1988), Bak (1996), and Kauffman (1995) for the details on self-organized criticality and its intimate connection with complex behavior). It has been argued that a complex adaptive system can thrive and display emergent robustness at the edge of chaos/order, just by employing a few good rules which provide the right moderate amount of flexibility (Kauffman, 1995). Too much flexibility based on a lot of rules can lead to chaos and destruction via an unexpected and sudden breakdown, whereas no flexibility often leads to ossification and rigidity. To the extent that a supply chain can be envisaged as a complex adaptive system [Surana et al. (2005) and Pathak et al. (2007)] it would be expected to achieve flexibility and robustness via the use of a few simple yet ingenious rules. Haksöz (2013) coins such supply chains as risk intelligent. Simple rules invoked under right contexts increase risk intelligence. Moreover, the value of these simple rules is shown to increase as the context where a supply chain operates becomes more uncertain and dynamic.

\section{B. How to allocate work within the supplier portfolio?}

Let us say, that a manufacturer has indeed identified two particular suppliers. Then, the question is what proportion of work should be allocated to each one of them.
This problem can be modeled as one of optimization. This approach was taken by Nobel laureate Harry Markowitz for a similar problem, the allocation of wealth to assets in a financial portfolio (Markowitz, 1952). For this problem, a simpler alternative to optimization is the $1 / N$ diversification rule, which allocates an equal amount of wealth to each of $N$ assets (which have been fixed previously). The $1 / N$ rule has a long history. Rabbi Isaac bar Aha recommended it in the Talmud in about the fourth century: "A man should always place his money, a third into land, a third into merchandise, and keep a third at hand" (Benartzi and Thaler, 2001).

The $1 / N$ rule does not rely on any mathematical theory. And it does not rely on any historical data about the previous performance of assets. Possibly because of these features of the $1 / N$ rule, researchers often view it as naïve, or even confused, and have, for example, concluded that "it does not assure sensible or coherent decision making" (Benartzi and Thaler, 2001).

Nevertheless, things look different when the performance of Markowitz's optimization model is compared empirically with that of the naïve $1 / N$ rule. It was recently found in computer simulations using real financial data that Markowitz's model - and 12 of its sophisticated Bayesian modifications-could not outperform the $1 / N$ rule in terms of standard measures such as return (measured by its certainty equivalent) and Sharpe ratio (average return in excess over the risk-free asset, divided by the standard deviation of the return) (DeMiguel et al., 2009). More specifically, the $1 / N$ rule ranked first among all models in certainty equivalent return and fifth in Sharpe ratio.

The main reason for this result is that the $1 / N$ rule does not require any data to run, whereas Markowitz's model requires ample high-quality data. It was estimated that Markowitz's model would need 250 years of historical data to outperform the $1 / N$ rule given a portfolio of 25 stocks for sample-based mean-variance policy (DeMiguel et al., 2009, p. 1941).

The $1 / N$ rule can be easily adapted to supply chain risk management: "Allocate work equally among suppliers." This rule has some clear advantages such as eliminating the need for calculations while increasing the transparency and trust in ongoing buyer-supplier relationships. Theoretical research has also found quantitative benefits of the $1 / N$ rule in supply chain risk management.

In practice, simple rules that approximate the equity of the $1 / \mathrm{N}$ rule are in use. For example, the manufacturing company Brisa uses two suppliers, which perform $60 \%$ and $40 \%$ of the work, respectively. The supply chain director explains their rationale as follows:

"We have long term relationships with raw material suppliers. We do not purchase in the spot market. We need approved suppliers to work with. Once we have a number of suppliers, we use a dual sourcing strategy. That is, we procure $60 \%$ from supplier A and $40 \%$ from supplier B in order to manage supplier related risks. Our main goal is to establish fruitful long term strategic alliances with suppliers, not just procure for only a few years" (Haksöz, 2013).

This concern with establishing relationships with suppliers that can be sustained for a long time underlies another 
rule of thumb for allocating work within a supplier portfolio. Before we state the rule, note first that the fact that a buyer requests a supplier to meet, say, $50 \%$ of its demand does not mean that the supplier can indeed meet this demand. Or perhaps it can, but only barely or if other contracts are modified or breached [Haksöz and Seshadri (2007) and Haksöz and Kadam (2009)] all of which outcomes are not desirable for any party. The supplier needs to get a big enough contract, but not too big, so that s/he can have the possibility of taking on other customers. Li and Fung, a global orchestrator of supply chains for thousands of suppliers and customers worldwide, use the following simple rule: "The work you allocate to each one of your suppliers should be between $30 \%$ and $70 \%$ of their capacity" (Fung et al., 2008).

This simple rule of thumb increases the robustness of Li and Fung and its suppliers. The upper bound of $70 \%$ allows the suppliers to maintain relationships with other buyers and reduces overly strong mutual dependency. The lower bound of $30 \%$ sustains the investment of effort by both parties. According to Li and Fung, these numerical bounds are not computed by using methods of optimization but are arrived at based on a process of intuition (Simon, 1990), which is formed by the experience and feedback Li and Fung executives have accumulated during many years of intimate work experience with thousands of diverse suppliers. Surely, these upper and lower bounds will change based on the type of supply chain and industry under focus. We use these bounds $(30 \%-70 \%)$ in this simple rule for only illustrative purposes.
The three simple rules presented in this section-use multiple or dual sourcing, allocate work equally among suppliers, and reserve between $30 \%$ and $70 \%$ of each supplier's capacity — can be combined in a graphical aid for diagnosing and managing supply chain risk, which is easy to understand and use. The aid is shown in Fig. 1.

This aid has the form of a fast and frugal decision tree (Martignon et al., 2008). In this article, decision trees are tree-like graphical representations of processes for making decisions. They have two kinds of nodes: input nodes in which information about the problem at hand is asked and output nodes in which, on the basis of the information provided previously, a decision or action is recommended. In fast and frugal decision trees, there is always at least one output node following each input node. This feature helps to achieve speedy decision making based on a few pieces of information, possibly even only one.

The tree in Fig. 1 queries managers on their use of the three rules and recommends appropriate actions according to their responses. If there is only a single supplier, this is highly risky for the robustness of the supply chain, and the manager is urged to identify additional suppliers. Note that appropriately the recommendation box is colored red (dark grey in print). If multiple or dual sourcing is used, the manager is next queried on the other two rules, allocation of work to suppliers and reservation of their capacity. If either of these rules is violated, the corresponding recommendation is made to the manager, albeit with less urgency and with recommendation boxes accordingly colored towards yellow

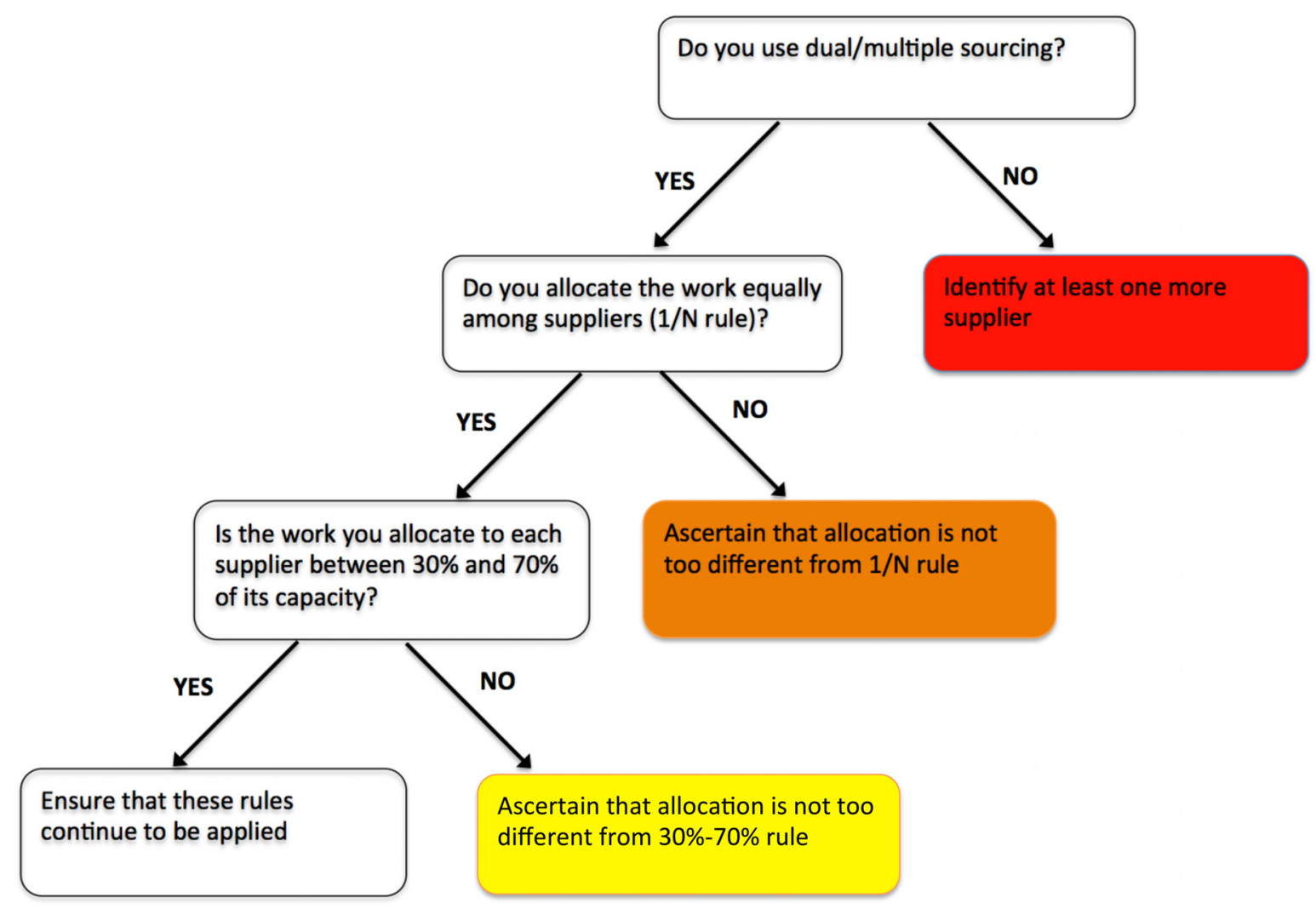

FIG. 1. A fast and frugal decision tree for diagnosing and managing supply chain risk, using the three simple rules discussed in this section. The manager should go through this aid repeatedly until ensuring that all three rules are in use. Note that the particular numbers in the (30\%-70\%) rule are presented only for illustration. They will vary for every supply chain. 
(lighter grey in print). The manager should go through the tree repeatedly until ensuring that all three rules are in use.

\section{WHEN SHOULD DECISION MAKERS USE FEW RESOURCES? EVIDENCE AND THEORY}

\section{A. Examples from practice}

Considering only one, two, or a few attributes to make an effective decision is not unique to supply chain risk management or the construction of financial portfolios. In this section, we aim to review examples from practice in different arenas of decision making.

In marketing, in order to decide whether to send advertising materials to a past customer, the amount of time since the customer's most recent purchase can be taken into account. In some industries, nine months without a purchase are often considered sufficient for declaring a customer inactive. It has been empirically found that this simple hiatus rule makes more accurate predictions of future sales than does a typical forecasting model, the Pareto/negative binomial distribution model, namely, $77 \%$ vs. $74 \%$ in an airline, and $83 \%$ vs. $75 \%$ in an apparel company, respectively (Wuebben and von Wagenheim, 2008).

Second, economists at the Bank of England, in collaboration with two of us, recently developed a fast and frugal tree for allowing regulators to monitor whether a bank is at high risk of bankruptcy or not (Aikman et al., 2014). Similar to the tree in Fig. 1, this tree uses four attributes in a simple sequential way (i.e., leverage ratio in the balance sheet, market-based capital ratio, total amount of wholesale funding, and loan to deposit ratio). In the dataset of 116 banks, which had more than 100 billion USD in assets at the end of 2006 , the tree correctly identified $82 \%$ of the banks that subsequently failed and $50 \%$ of the banks that did not fail. The fast and frugal tree was not outperformed by any of 20 versions of the usual tool of financial economics, logistic regression-which used the same economic indicators as the tree-while being much easier to understand and use.

As a final example, consider a key aspect of modern stability operations (e.g., in Afghanistan or Kosovo), the number of civilian casualties. Such operations aim at creating a stable and secure environment that allows for the creation of democratic institutions. Beyond being morally imperative, the minimisation of civilian casualties is a central strategic concern in stability operations. Any loss of life on the part of the civilian population not only increases local resentment, swelling the ranks of resistance forces, but is also a direct failure to fulfil the political mandate of the operation.

Consequently, there has been increasing pressure to minimise civilian casualties at NATO presences, such as checkpoints (Petraeus, 2009). In some types of operations in which the decision to use force has to be approved by higher levels of command, as in close air support, the civilian death toll has decreased. Yet, in situations in which individual soldiers have to, by themselves, assess potential threats and judge how to react appropriately under time pressure, no such reductions have been forthcoming. This is the case in a NATO checkpoint where a vehicle is approaching and the soldier staffing the checkpoint has to quickly decide if, when, and how exactly to escalate force so that the vehicle stops and collaborates in the inspection process.

It is not clear how to apply the usual analytical models to this problem. The data required by standard decision trees are not available. For example, in a dataset including 1060 incident reports of situations involving motor vehicles approaching a NATO military presence between January 2004 and December 2009 in Afghanistan, there were only seven suicide attacks recorded. These seven reports discuss only the battle damage and provide little information which could be used for predicting hostile incidents in the future such as the characteristics of the hostile vehicle. Thus, it is not clear how to solve this problem with standard methods of optimization: The only decision tree that can be induced from the dataset of 1053 nonhostile incidents is one that predicts that an approaching vehicle is never hostile and this will obviously not do. The seven incidents in which suicide attackers were missed may be added to the dataset but the issue then is that only the attribute of the number of occupants in the vehicle is available in these reports; then, the induced decision tree would suggest that a vehicle is always hostile when there is one occupant, which would result in many false alarms and likely civilian casualties.

Alternatively, a fast and frugal decision tree can be constructed by using methods of cognitive field research such as reviews of the academic and practitioner literature, observations of military training, and interviews with armed forces instructors and other experienced personnel. This was done in a project involving one of us and researchers and staff from the German Armed Forces (Keller and Katsikopoulos, 2016). A fast and frugal decision tree was proposed for classifying a vehicle approaching a checkpoint as hostile or not. The tree used only three attributes: the number of occupants in the vehicle, whether the vehicle slows down or not while approaching the checkpoint, and available intelligence information. Had the tree been applied to NATO checkpoints in Afghanistan between 2004 and 2009, the number of civilian casualties would have been reduced from 204 to 78, while terrorist attacks would be detected at least as often as when soldiers decided without the help of the tree.

\section{B. Review of the evidence}

The few examples discussed above suggest that simple rules - such as using only one attribute or all attributes but without weighting them as in a $1 / N$ rule-may be able to compete with more complex models. But are these just isolated incidents?

A fair amount of empirical evidence has accumulated on the comparison between simple rules and more complex models typically employed in operations research, statistics, and computer science, such as regressions (i.e., linear, logistic, and regularized), Bayesian networks (e.g., naïve Bayes), neural networks, classification and regression trees, and support vector machines (Katsikopoulos, 2011). The problems studied in this research include multi-attribute choice, classification, and forecasting and belong to domains as diverse as economics, transportation, and healthcare. Below, we review 
studies that used multiple real world datasets to compare the performance of simple rules and more complex models.

The first such study was performed in 1999 and measured the performance of linear regression, a one-attribute rule, and a $1 / N$ rule on 20 datasets from the domains of biology, environmental science, demography, health, psychology, and transportation (Czerlinski et al., 1999). The average number of alternative options in a dataset was 67. In each dataset, all multi-attribute choices involving two out of a fixed half of the alternatives in the dataset were constructed and used as the set on which the models were trained. The performance of the models, with their parameters fixed at the values estimated from the training set, was measured on the other half of the dataset, which is called the test set. An example of model parameters is the weights of the attributes used in a linear regression. Predictive performance was measured as the percentage of agreement between model predictions and ground truth in the test set. Say that a choice is to pick one out of two American high schools with the higher dropout rate; choosing the high school that indeed has the higher rate agrees with the ground truth. This procedure was repeated 1000 times in order to use different training and test sets and thus average out random variation.

The main finding of this study is that, averaged across datasets, the predictive accuracy of the one-attribute rule equals that of linear regression, $76 \%$, whereas that of the $1 / \mathrm{N}$ rule is $69 \%$. This result has been replicated and extended to include other complex models (e.g., naïve Bayes) and very small training sets (i.e., up to $15 \%$ of the whole dataset), which may be more realistic for decision making under changing conditions [Katsikopoulos (2011) and Czerlinski et al. (1999)]. For very small training sets, the one-attribute rule outperforms naïve Bayes by an average of $5 \%$ in predictive accuracy.

Second, the performance of classification and regression trees, logistic regression, and two versions of fast-and-frugal trees was tested on 30 datasets from the UC Irvine Machine Learning Repository (Martignon et al., 2008). All datasets referred to classifications, with 11 of them referring to medical classifications. Training set sizes of $85 \%, 50 \%$, and $15 \%$ of the whole datasets were used. The main finding is a strong effect of training set size. The best complex model outperforms the best simple rule for the largest training set by $4 \%$ ( $82 \%$ vs. $78 \%$ ), but the difference shrinks to $1 \%$ (76\% vs. $75 \%$ ) for the smallest training set.

In a third study looking at a broad range of problems, 51 datasets were used spanning the domains of biology, business, computer science, ecology, economics, education, engineering, environmental science, medicine, political science, psychology, sociology, sports, and transportation (Şimşek, 2013). A stateof-the-art version of linear regression with elastic net regularization was compared with a one-attribute rule. The size of the training set was equal to that of the whole dataset minus one. The main finding is that regularized linear regression scores $79 \%$ and the one-attribute rule $78 \%$.

On the basis of this evidence, there appear to be no large differences in accuracy between simple rules of thumb and more complex models. This can be explained by the flat maximum effect (Lovie and Lovie, 1986). What this effect says is that the attribute weights used in a linear model do not greatly change the overall deviation between true and forecasted values. The flat maximum effect is relevant because many of the simple rules and more complex models tested can indeed be viewed as linear models (Katsikopoulos, 2011).

On the other hand, it is also clear that even a difference in accuracy of $1 \%$ can have enormous implications in some contexts, as when the customer base of a multi-national company includes hundreds of thousands or even millions of people, or in retail settings where profit margins are razor thin. Thus, it is necessary to have a theory delineating the general conditions under which simple rules perform better than more complex models, and vice versa [Hogarth and Karelaia (2005), Hogarth and Karelaia (2006), and Katsikopoulos and Gigerenzer (2013)].

\section{Review of the theory}

A general framework for understanding the comparative performance of simple rules and more complex models is provided by the statistical theory of prediction, and, in particular, the bias-variance decomposition of prediction error [Geman et al. (1992) and Gigerenzer and Brighton (2009)].

This decomposition is a mathematical fact which says that the prediction error of a model, be it simple or complex, is the sum of two terms. The first term is called bias and it measures how well, on the average, the model agrees with the ground truth. Complex models-which usually have many parameters-tend to have less bias than simple models - which usually have fewer parameters-because when parameters can be tweaked, the agreement between model prediction and ground truth can increase as well. For example, Markowitz's multi-parameter optimization model achieves low bias, whereas the $1 / N$ rule has zero parameters and has relatively high bias.

But this is not the whole story. There is a second term, called variance, which contributes to a model's total prediction error. Variance measures the variation of model predictions around the model's average prediction. Unlike the bias term, when it comes to the variance term, model complexity is less of a blessing and more of a curse. Complex multiparameter models tend to have higher variance than simple models with fewer parameters, because more parameters can combine in more ways and generate more distinct predictions.

For example, one can intuit why, when the training set size is small, simple rules tend to have lower variance than more complex models. The smaller the training set, the more likely it is that sampling error and natural variations in the instances which are included in the training set will lead to variation in the parameter estimates of a given model. This variation can be expected to have an influence on the more heavily parameterized models to a greater degree than on the simpler rules. In an extreme case, Markowitz's multiparameter optimization model has relatively high variance, whereas the $1 / N$ rule has zero variance because it has zero parameters.

Because a model's total prediction error is the sum of its bias and variance, one can see that the result can go either way: A simple or a more complex model can have higher 
predictive accuracy in a particular dataset, depending on whether an advantage in bias is larger than an advantage in variance in this dataset.

It has been argued that, in practice, variance may play a more critical role than bias (Brighton and Gigerenzer, 2015). This claim is consistent with a recent review of the forecasting literature which concluded that all valid evidence-based forecasting methods are simple, and urged decision makers to only accept forecasts from simple methods (Green and Armstrong, 2015).

Surprisingly, it has been recently discovered that simple rules may also achieve competitive bias in practice. This happens when there exists an attribute, or an alternative option, which dominates the others.

An attribute dominates other attributes when it is subjectively much more important to the decision maker than the other attributes. For example, supplier responsiveness or cost efficiency may be much more important to a particular buyer than other supplier attributes. A second meaning of attribute dominance is when an attribute is statistically much more informative of the utility of options than other attributes. For instance, time since last purchase predicts future sales much more accurately than customer age does. It has been analytically shown that a one-attribute rule that uses a dominant attribute incurs zero bias (Katsikopoulos, 2011).

An alternative option dominates other options when its attribute values are better or equal to the attribute values of the other options. It should be noted that less restrictive definitions of dominance exist that also lead to good or even theoretically optimal performance of simple rules of thumb (Baucells et al., 2008). Assuming that utility is an additive or multi-linear function of the attributes, it has been analytically shown that some one-attribute and $1 / N$ rules have zero bias when a dominant alternative exists.

One may think that dominant attributes and alternatives are rare in the real world. In fact, the opposite seems to be the case (Şimşek, 2013). Across 51 real datasets, it was found that dominant attributes exist in $93 \%$ of binary datasets (i.e., attributes had values of 1 or 0 ) and in $83 \%$ of the numeric datasets, and that dominant alternatives exist in $87 \%$ and $58 \%$ of binary and numeric datasets, respectively.

In sum, the conclusion of the theoretical work is that simple rules of thumb tend to perform better than more complex models when $(i)$ the information available is not of high quality or not ample enough to estimate the parameters of models reliably or (ii) there exists one attribute, or one alternative option, which dominates the others. On the other hand, when neither of conditions (i) nor (ii) hold, complex models tend to perform better than simple rules of thumb.

Condition (i) essentially says that a problem is difficult. Such difficulties may arise when a problem is dynamic or future developments are unpredictable. Both issues are present in the problem of constructing a financial or global supply portfolio [(Benartzi and Thaler, 2001), (Haksöz and Seshadri, 2007), and (Haksöz, 2013)]. If (i) holds, an advantage in the variance component of the prediction error can be expected to be much larger than the bias component, and simple rules have a very good chance of outperforming more complex models.

An interesting interpretation of condition (ii) is that it says that the problem is easy, in the following sense. There either exists one alternative option which is better than all other options and the decision maker only needs to realize this and identify this option or there exists one attribute which is so important or informative that it suffices to only consult this attribute and, again, the decision maker only needs to realize this and identify this option. If (ii) holds, as empirical research has shown that it often does in practice, several simple rules achieve zero bias, and thus can indeed outperform more complex models.

A summary of the empirical and theoretical results on the general conditions under which simple rules of thumb outperform more complex models and vice versa is provided in Table I.

\section{A FOUR-STEP PROCESS FOR DETERMINING THE AMOUNT OF RESOURCES TO USE}

On the basis of the evidence and theory discussed in Sec. III-summarized in Table I-we recommend a fourstep process for determining the amount of resources decision makers should use:

(1) Ensure that your company does not decide defensively (e.g., "we have always used this software, for which we pay expensive technical support" or "we have always trusted our gut feelings") but remains open to adopting either simple rules or more complex models, depending on the problem at hand.

(2) Identify the problem's type: Is the problem difficult, easy, or neither? (See the text preceding Table I for definitions of these terms).

TABLE I. A summary of the empirical evidence and theoretical analyses on the general conditions under which simple rules of thumb outperform more complex models and vice versa, as discussed in this section.

\begin{tabular}{lr}
\hline \hline $\begin{array}{l}\text { Approach to decision making } \\
\text { Simple rules of thumb }\end{array}$ & Types of problems for which each approach tends to perform better \\
$\begin{array}{l}\text { (e.g., Use only one attribute; use a few attributes as in fast and frugal trees; } \\
\text { use all attributes but do not weight them differently, as in a } 1 / N \text { rule) }\end{array}$ & (e.g., Low-quality or scant information, dynamic or unpredictable situations); \\
Easy problems & (i.e., problems with dominant attributes or dominant alternative options) \\
$\begin{array}{l}\text { More complex models, typically employed in operations research, } \\
\text { statistics and computer science } \\
\text { (e.g., Linear and logistic regression, Bayesian networks, neural networks, } \\
\text { classification and regression trees) }\end{array}$ & $\begin{array}{c}\text { Other problems } \\
\text { lems without dominant attributes or dominant alternative options) }\end{array}$ \\
\hline
\end{tabular}


(3) Consult Table I to choose a simple rule or a complex model, which is expected to perform best for your problem's type.

(4) Record the performance of the model you chose and use it to possibly update Table I for the domain and problem in which you work.

In sum, against the recent trend of focusing on resourceintensive models, the results presented herein suggest that there are good reasons for considering simple rules of thumb.

\section{CONCLUSION}

How novel is the message of the merit of simplicity in supply chain management? It has been put forward in other areas of management such as strategy (Eisenhardt and Sull, 2001) and is entertained in related fields such as systems thinking and "soft" operations research (Rosenhead and Mingers, 2001). The main difference is that the simple rules discussed in this article do not apply to so-called wicked or messy problems with unclear objectives or multiple disagreeing stakeholders. The simple rules presented here are quantitative and apply to well-defined problems of performance on set operational objectives where success can be measured; they are not qualitative principles for clarifying or prioritizing objectives. For example, operational performance of a simple rule of dual sourcing in the context of supply chain risk can be measured quantitatively and the flexibility and robustness it provides can be demonstrated. Supplier portfolio allocation is another such well-defined problem addressed via a proposed simple rule of $1 / \mathrm{N}$. Whereas sometimes simple rules may focus on counteracting biases (Tiwana et al., 2007), the rules presented here focus on how effective decisions are made.

On the other hand, the process of deriving the various simple rules is to a large extent common across fields such as supply chain management, operations research, and business strategy. It is typically based on the observation and analysis of people's purposeful behavior (Simon, 1990). In this sense, the simple rules of thumb we discussed may be viewed as expert systems (Jackson, 1986). Now, it is important to note that expertise has not always been associated with simplicity. The results presented here show that knowing when to go for simplicity may well be an important part of expertise. Today's decision makers need to remain open to how simplicity can be the key to flexibility and robustness.

\section{ACKNOWLEDGMENTS}

The authors are grateful to the editorial team and referees for their helpful suggestions and comments, which have improved the quality of the paper. The research of Çağrı Haksöz is supported by Sabancı University, Max Planck Institute for Human Development, and DAAD Scholarship.

Aikman, D., Galesic, M., Gigerenzer, G., Kapadia, S., Katsikopoulos, K. V., Kothiyal, A., Murphy, E., and Neumann, T., "Taking uncertainty seriously: Simplicity versus complexity in financial regulation," Bank of England Financial Stability Paper No. 28, 2014.

Allon, G. and Van Mieghem, J. A., "Global dual sourcing: Tailored basesurge allocation to near- and offshore production," Manage. Sci. 56(1), 110-124 (2010).
Bak, P., How Nature Works: The Science of Self-Organized Criticality (Copernicus, New York, 1996).

Bak, P., Tang, C., and Wiesenfeld, K., "Self-organized criticality," Phys. Rev. A. 38(1), 364-375 (1988).

Baucells, M., Carrasco, J. A., and Hogarth, R. M., "Cumulative dominance and heuristic performance in binary multiattribute choice," Oper. Res. 56(5), 1289-1304 (2008).

Benartzi, S. and Thaler, R. H., "Naive diversification strategies in defined contribution saving plans," Am. Econ. Rev. 91(1), 79-98 (2001).

Brighton, H. and Gigerenzer, G., "The bias bias," J. Bus. Res. 68, 1772-1784 (2015).

Czerlinski, J., Gigerenzer, G., and Goldstein, D. G., "How good are simple heuristics?," in Simple Heuristics That Make Us Smart, edited by Gigerenzer, G., Todd, P. M., and ABC Research Group (Oxford University Press, New York, NY, 1999), pp. 97-118.

DeMiguel, V., Garlappi, L., and Uppal, R., "Optimal versus naïve diversification: How inefficient is the $1 / \mathrm{N}$ portfolio strategy?," Rev. Financ. Stud. 22(5), 1915-1953 (2009).

Eisenhardt, K. M. and Sull, D. N., "Strategy as simple rules," Harv. Bus. Rev. 79(1), 106-116 (2001).

Fu, Q., Lee, C. Y., and Teo, C. P., "Procurement management using option contracts: Random spot price and the portfolio effect," IIE Trans. 42, 793-811 (2010).

Fung, V. K., Fung, W. K., and Wind, Y., Competing in a Flat World: Building Enterprises for a Borderless World (Wharton School Publishing, Philadelphia, PA, 2008).

Geman, S., Bienenstock, E., and Doursat, E., "Neural networks and the bias/ variance dilemma," Neural Comput. 4(1), 1-58 (1992).

Gigerenzer, G. and Brighton, H., "Homo heuristicus: Why biased minds make better inferences,” Top. Cognit. Sci. 1(1), 107-143 (2009).

Green, K. C. and Armstrong, J. S., "Simple versus complex forecasting: The evidence,” J. Bus. Res. 68(8), 1678-1685 (2015).

Greimel, H., "Freescale seeks Japan toehold with motorsports marketing," Automotive News 88(6610), 24 (2014).

Haksöz, Ç., Risk Intelligent Supply Chains (Taylor and Francis, Boca Raton, FL, 2013).

Haksöz, Ç. and Kadam, A., "Supply portfolio risk," J. Oper. Risk 4(1), 59-77 (2009).

Haksöz, Ç. and Seshadri, S., "Supply chain operations in the presence of a spot market: A review with discussion," J. Oper. Res. Soc. 58(11), 1412-1429 (2007).

Heuristics: The Foundations of Adaptive Behavior, edited by Gigerenzer, G., Hertwig, R., and Pachur, T. (Oxford University Press, Oxford, UK, 2011).

Hogarth, R. M. and Karelaia, N., "Simple models for multiattribute choice with many alternatives: When it does and does not pay to face tradeoffs with binary attributes?," Manage. Sci. 51, 1860-1872 (2005).

Hogarth, R. M. and Karelaia, N., "Regions of rationality: Maps for bounded agents," Decis. Anal. 3(3), 124-144 (2006).

Jackson, P., Introduction to Expert Systems (Addison Wesley, Reading, MA, 1986).

Katsikopoulos, K. V., "Psychological heuristics for making inferences: Definition, performance and the emerging theory and practice," Decis. Anal. 8(1), 10-29 (2011).

Katsikopoulos, K. V. and Gigerenzer, G., "Behavioral operations management: A blind spot and a research program," J. Supply Chain Manage. 49, 3-7 (2013).

Kauffman, S., At Home in the Universe: The Search for the Laws of SelfOrganization and Complexity (Oxford University Press, Oxford, UK, 1995).

Keller, N. and Katsikopoulos, K. V., "On the role of psychological heuristics in operational research; and a demonstration in military stability operations," Eur. J. Oper. Res. 249, 1063-1073 (2016).

Lovie, A. D. and Lovie, P., "The flat maximum effect and linear scoring models for prediction," J. Forecasting 5(3), 159-168 (1986).

Markowitz, H., "Portfolio selection,” J. Finance 7(1), 77-91 (1952).

Martignon, L., Katsikopoulos, K. V., and Woike, J. K., "Categorization with limited resources: A family of simple heuristics," J. Math. Psychol. 52(6), 352-361 (2008).

Pathak, S. D., Day, J. M., Nair, A., Sawaya, W. J., and Kristal, M. M., "Complexity and adaptivity in supply networks: Building supply network theory using a complex adaptive systems perspective," Decis. Sci. 38(4), 547-580 (2007)

Petraeus, D., Tactical directive 100804, Headquarters of the International Security Assistance Force, Kabul, Afghanistan, 2009, see http://www. 
isaf.nato.int/article/isaf-releases/general-petraeus-issues-updated-tacticaldirective-emphasizes-disciplined-use-of-force.html (last accessed April 14, 2016).

Rosenhead, J., "Robustness analysis," in Encyclopedia of Operations Research and Management Science (Springer, New York, NY, 2013), pp. 1346-1347.

Rational Analysis for a Problematic World Revisited: Problem Structuring Methods for Complexity, Uncertainty and Conflict, edited by Rosenhead, J. and Mingers, J. (Wiley, New York, NY, 2001).

Simon, H., "Invariants of human behavior," Annu. Rev. Psychol. 41, 1-20 (1990).

Şimşek, Ö., "Linear decision rule as aspiration for simple decision heuristics," in Advances in Neural Information Processing Systems, edited by
Burges, C. J. C., Bottou, L., Welling, M., Ghahramani, Z., and Weinberger, K. Q. (2013), Vol. 26, pp. 2904-2912.

Surana, A., Kumara, S., Greaves, M., and Raghavan, U. N., "Supply-chain networks: A complex adaptive systems perspective," Int. J. Prod. Res. 43(20), 4235-4265 (2005).

Tang, C. and Tomlin, B., "The power of flexibility for mitigating supply chain risks," Int. J. Prod. Econ. 116, 12-27 (2008).

Tiwana, A., Wang, J., Keil, M., and Ahluwalia, P., "The Bounded rationality bias in managerial valuation of real options: Theory and evidence from IT projects," Decis. Sci. 38(1), 157-181 (2007).

Wuebben, M. and von Wagenheim, F., "Instant customer base analysis: Managerial heuristics often 'get it right'," J. Mark. 72(3), 82-93 (2008). 